\title{
Raster-elektronenmikroskopische Studien an Pollen aus Bienenhonig. III. Der Harmomegathie-Mechanismus und seine Auswirkungen auf die Exine-Strukturen am Beispiel unterschiedlicher Pollentypen
}

\author{
K von der Ohe, JH Dustmann \\ Niedersächsisches Landesinstitut für Bienenkunde, Wehlstrasse 4 a, D-3100 Celle, BRD
}

(eingegangen 25 November 1989; angenommen 4 Juni 1990)

\begin{abstract}
Zusammenfassung - Pollen im Honig unterliegen einer Volumen- und Formveränderung durch Wasseraufnahme des Plasmas. Der hierdurch bedingte sogenannte Harmomegathie-Mechanismus wurde an unterschiedlichen Exinemustern tricolpater Pollen beobachtet. Um die morphologischen Unterschiede an kontrahierten und expandierten Pollen zu erfassen, wurde ein REM eingesetzt. Die Veränderungen der Ektexine der Intercolpien zeigen, daß bei der Erweiterung des Pollenumfanges eine auffällige Biegung der Exine stattfindet, welche die Oberflächenstruktur erheblich beeinflußt. Bei reticulater Anordnung ist eine Stauchung der Skulpturelemente deutlich sichtbar.
\end{abstract}

Pollen / Honig / Harmomegathie / Exinestruktur / REM

\section{EINLEITUNG}

Mit dem Aufplatzen der Antheren werden Pollen extrem veränderten mikroklimatischen Verhältnissen ausgesetzt: Im Gegensatz zu dem Stadium der Reifung in der Anthere befinden sie sich nach deren Aufplatzen in einem feuchtigkeitsarmen Medium. Das Protoplasma muß jedoch vor einer Dehydration geschützt werden. In der kurzen Zeitspanne des Wasserverlustes verändert der Pollen sein Volumen. Diesen Vorgang der Volumenänderung durch Feuchtigkeitsabgabe bzwaufnahme bezeichnete Wodehouse (1935) mit dem Terminus "Harmomega- thy". Mit der Volumenänderung geht eine z T extreme Formveränderung einher. Sie ist von der Struktur der Pollenkornwand abhängig, d h von der Art und Anzahl der Aperturen. So werden bei Wasserverlust die Colpusränder nach innen eingezogen und verschließen damit die Pollenkornwand.

In diesem kontrahierten Zustand befindet sich das Pollenkorn unter Stress (hier: Maß für den negativen Druck auf die Pollenkornwand). In einem Pollenkorn im Stress-Zustand befindet sich das Protoplasma nach Feuchtigkeitsverlust im Äquilibrium mit der dehydrierenden Atmosphäre (Payne, 1981). 
Gelangt das Pollenkorn auf das Stigma der zu bestäubenden Blüte und damit in ein feuchtes Medium, findet erneut Harmomegathie statt: Aufgrund der Wasserabsorption entspannen sich die Pollenkornwände und die Aperturen öffnen sich, durch die der Pollenschlauch den plasmatischen Inhalt mit den generativen und vegetativen Kernen in das Ovar befördert (Punt, 1986; Iwanami et al, 1988).

Mit dem Phänomen der Harmomegathie haben sich Autoren in vielfältiger Weise auseinandergesetzt. Zander (1935) wies prinzipiell auf Trocken- und Quellformen der von inm untersuchten Pollen hin. Vorrangig wurden Volumenänderungen bestimmter Pollentypen (Payne, 1972) sowie Unterschiede der Harmomegathie-Mechanik aufgrund diverser Apertur-Typen (Payne, 1972, 1981; Blackmore und Barnes, 1985) analysiert. Bolick (1981) nahm statische und mechanische Gesetze zu Hilfe, um die Harmomegathie der Pollen zu erläutern.

Es stellte sich heraus, daß an der Volumenänderung nicht nur die Aperturen sondern auch die dazwischenliegende Exine beteiligt sind, und zwar mit einem ähnlichen Mechanismus wie er bereits für die Aperturen beschrieben worden war (Suarez-Cervera und Seoane-Camba, 1986).

Studien über Harmomegathie-Effekte sind auch in der Melissopalynologie von besonderem Interesse. Kontrahierte Pollen gelangen durch Wind oder Insekten in den Nektar, den die Honigbiene einträgt. In diesem Medium, das den Bedingungen auf dem Stigma der Blüte sehr ähnlich ist, kommt es ebenfalls zur Harmomegathie.

Pollenkörner im Honig liegen daher im keimungsfähigen, expandierten und somit entspannten Zustand vor. Wie bereits Payne (1972) den Harmomegathie-Effekt nutzte, um klare Abgrenzungen zwischen Porus und Colpus zu erlangen, kann der
Vergleich der strukturellen und skulpturellen Veränderung einer Pollenart zu Differenzierungszwecken herangezogen werden. So wurde die Möglichkeit aufgezeigt, mit Hilfe eines Diagramms - zusammengesetzt aus den Kriterien des kontrahierten sowie des expandierten Pollenkornes Unterscheidungsmerkmale bei den Pollen verschiedener Apfelsorten zu finden (Dustmann und Bote, 1985, 1987).

Diese Arbeit beschäftigt sich mit den Veränderungen der Skulpturierung auf den Intercolpien, $\mathrm{d}$ h weniger mit den Aperturen, sondern vor allem mit der dazwischenliegenden Exine. Die von Dustmann und Bote (1987) aufgestellte These, daß die Exine bei der Harmomegathie keine Dehnung, sondern lediglich eine Verbiegung mit einhergehender Stauchung von Skulpturelementen erfährt, soll hier durch die Beobachtung verschiedenartiger Skulpturtypen an expandierten Pollen bestätigt werden.

\section{MATERIAL UND METHODE}

Pollen mit unterschiedlichen Skulpturtypen wurden zur Analyse verwendet:

Ligustrum vulgare - Oleaceae

Leucophyllum frutescens - Scrophulariaceae

Fagopyrum esculentum - Polygonaceae

Acer platanoides - Aceraceae

Verbesina persicifolia - Compositae

Die Pollen der jeweiligen Blüten wurden mit Ether von den geplatzten Antheren gewaschen.

Die Präparation für die raster-elektronenmikroskopischen Analysen erfolgten an jeweils kontrahierten sowie expandierten Pollen.

REM-Analyse an kontrahierten Pollen : Die Pollen wurden auf einem mit beidseitigem Klebeband versehenen Aluminium-Probenhalter gestäubt und im Sputter-Coater PS-2 (ISI) mit einer $20 \mathrm{~nm}$ Goldschicht überzogen.

REM-Analyse an expandierten Pollen: Kontrahierte Pollen wurden in filtrierten Honig überführt, wo sie bei einer Temperatur von ca 
$35^{\circ} \mathrm{C}$ innerhalb von 48 Stunden expandierten. Nach Anwendung der Methode II (Dustmann und Bote, 1985) - Transfer in ein Edelstahlnetz, Entwässerung in Aceton und der aufsteigenden Ethanol-Reihe, Trocknung und Stabilisierung über den Kritischen Punkt von flüssigem Kohlendioxid (CPD-Apparatus E-3000, Polaron) - wurden die Pollen auf einen mit doppelseitigem Kletueband versehenen Aluminium-Probenhalter überführt und mit einer $20 \mathrm{~nm}$ Goldschicht versehen.

Alle Präparate wurden mit dem REM ISISuper-II bei einer Beschleunigungsspannung von $10 \mathrm{kV}$ analysiert. Die einzelnen Präparate wurden derart durchgesehen, daß eine Auswahl von repräsentativen Pollen für genauere Oberflächenstudien getroffen werden konnte. Zu diesem Zweck wurden Fotografien bei Vergrößerungen von $\times 2000$ und $\times 5000$ angefertigt. Die folgenden morphologischen Beschreibungen wurden nach der Terminologie von Faegri und Iversen (1964) und Erdtmann (1952) angegeben.
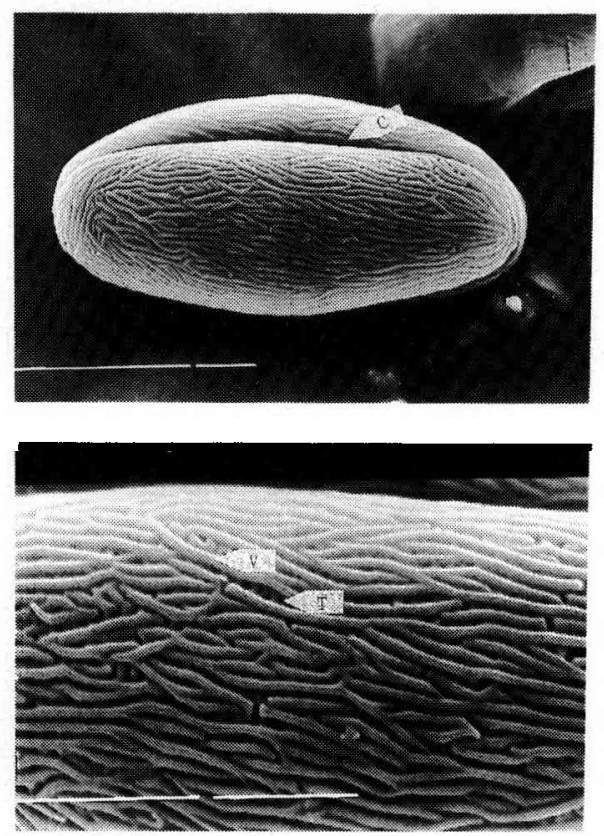

\section{BEOBACHTUNGEN}

Für das Studium der Skulpturierungen wurden colpate bzw colporate Pollen (Pollen mit Keimfalten bzw Keimfalten mit Poren) herangezogen. Pollen mit derartigen Harmomegathie-Elementen zeigen besonders deutlich die Veränderungen der Ektexine (äußere Schicht der Exine) des Intercolpiums. Es folgen die morphologischen Beschreibungen der verschiedenen Pollenarten sowie der Veränderungen durch Harmomegathie.

\section{Acer platanoides - Aceraceae (Abb 1)}

Die Pollen sind tricolpat. Als Skulpturelemente treten striate, longitudinal verlau-
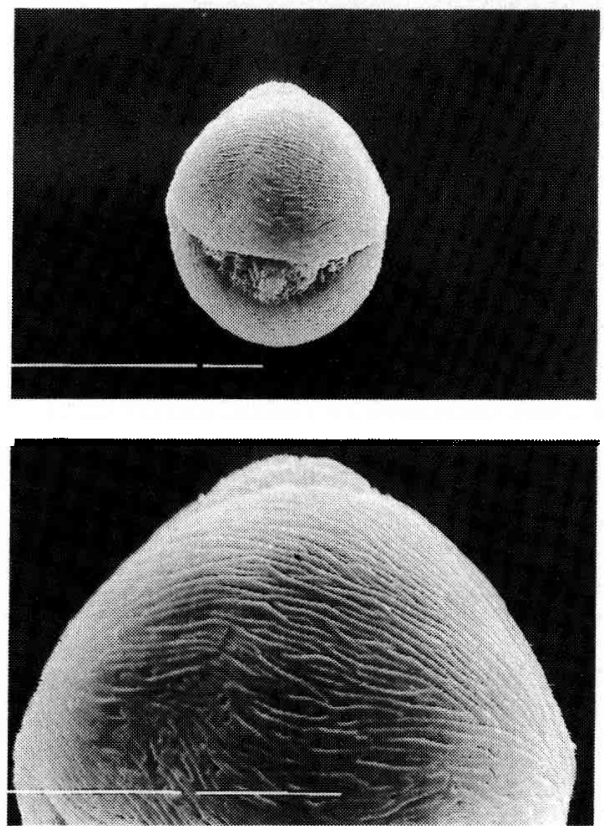

Abb 1. Pollen von Acer platanoides - Aceraceae. a) kontrahiert : Form prolat-oval, Colpi (C) eingezogen ( $\times 2000)$; b) expandiert : Form oval, spheroidal bis oblat, Colpi geöffnet $(\times 2000)$; c) kontrahiert : longitudinal verlaufende Vallae (V), darunterliegendes Tectum mit Perforationen $(T)$ sichtbar $(\times 5000)$; d) expandiert : Vallae verschließen das Tectum $(\times 5000)$; rechter $\mu$-marker $=5 \mu \mathrm{m}$. 
fende Valla (Wälle) hervor, die stellenweise verzweigt oder auch unterbrochen sind. In der kontrahierten Phase des Pollenkornes ist zwischen den Valla ein perforiertes Tectum (Basis-Schicht, auf der die Skulpturelemente aufgebaut sind) deutlich sichtbar, während in der expandierten Phase die Valla eng zusammengerückt erscheinen und das Tectum verschließen.

Die ovale Form des kontrahierten Pollenkornes ändert sich von prolat zu sphäroidal-oblat im expandierten Zustand.

\section{Ligustrum vulgare - Oleaceae (Abb 2)}

Die Pollen sind tricolporat. Die Ektexine zeigt reticulate (netzartige) Skulpturelemente. Die weiten Lumina (Zwischenräume) lassen die darunterliegenden
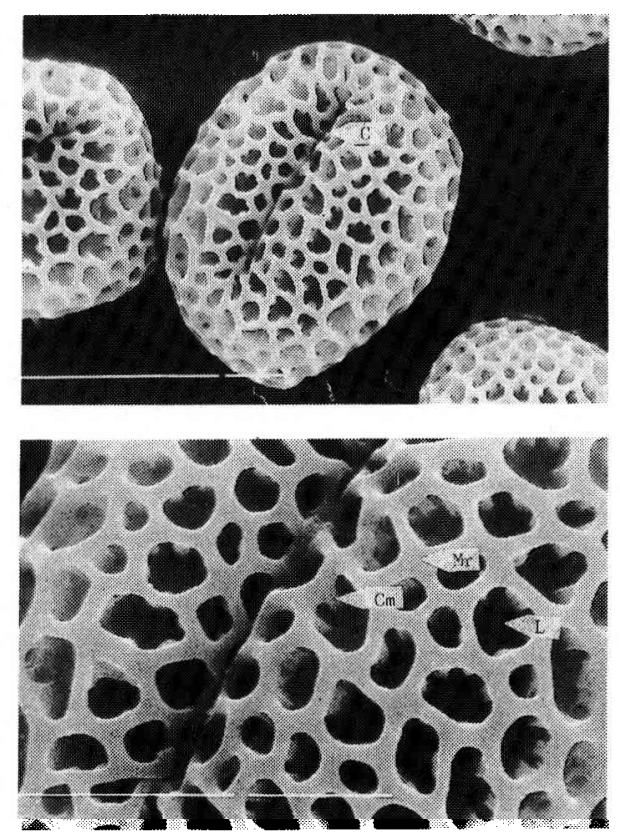

Columellae (Säulen) erkennen. Im Vergleich der Kontraktions- zur Expansionsphase ist eine deutliche Reduzierung der Lumina-Weite zu erkennen. Eine Verringerung des Abstandes zwischen den Columellae ist feststellbar.

Das ovale Pollenkorn liegt im kontrahierten Zustand in einer prolaten, nach der Expansion in einer sphäroidalen Form vor.

\section{Leucophyllum frutescens - Scrophulariaceae (Abb 3)}

Die Pollen sind tricolporat, wobei jeder Colpus eine Endexine (innere Schicht der Exine) mit zwei porenähnlichen Aperturen aufweist.

Die Ektexine zeigt eine unregelmäßig reticulate Skulpturierung mit - im Gegen-
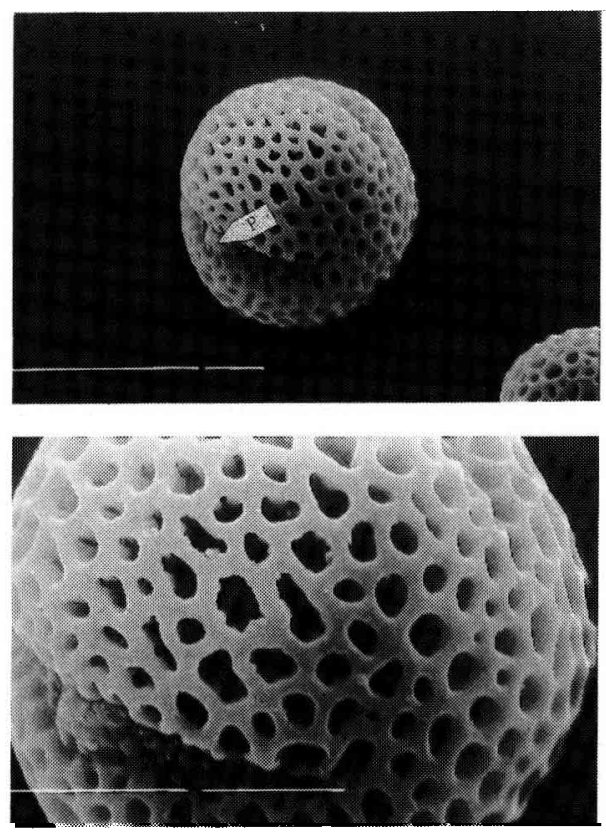

Abb 2. Pollen von Ligustrum vulgare - Oleaceae. a) kontrahiert : Form prolat-oval, Colpi (C) eingezogen $(x 2000)$; b) expandiert : Form sphäroidal-oval, Colpi geöffnet, endoaperturale Pore (P) sichtbar ( $x 2000)$; c) kontrahiert : reticulat angeordnete Muri (Mr) bilden weite Lumina (L), Columellae (Cm) sichtbar (x 5000 ); d) expandiert : Lumina-Weite und Abstand zwischen den Columellae verringert ( $x$ $5000)$; Rechter $\mu$-Marker $=5 \mu \mathrm{m}$. 

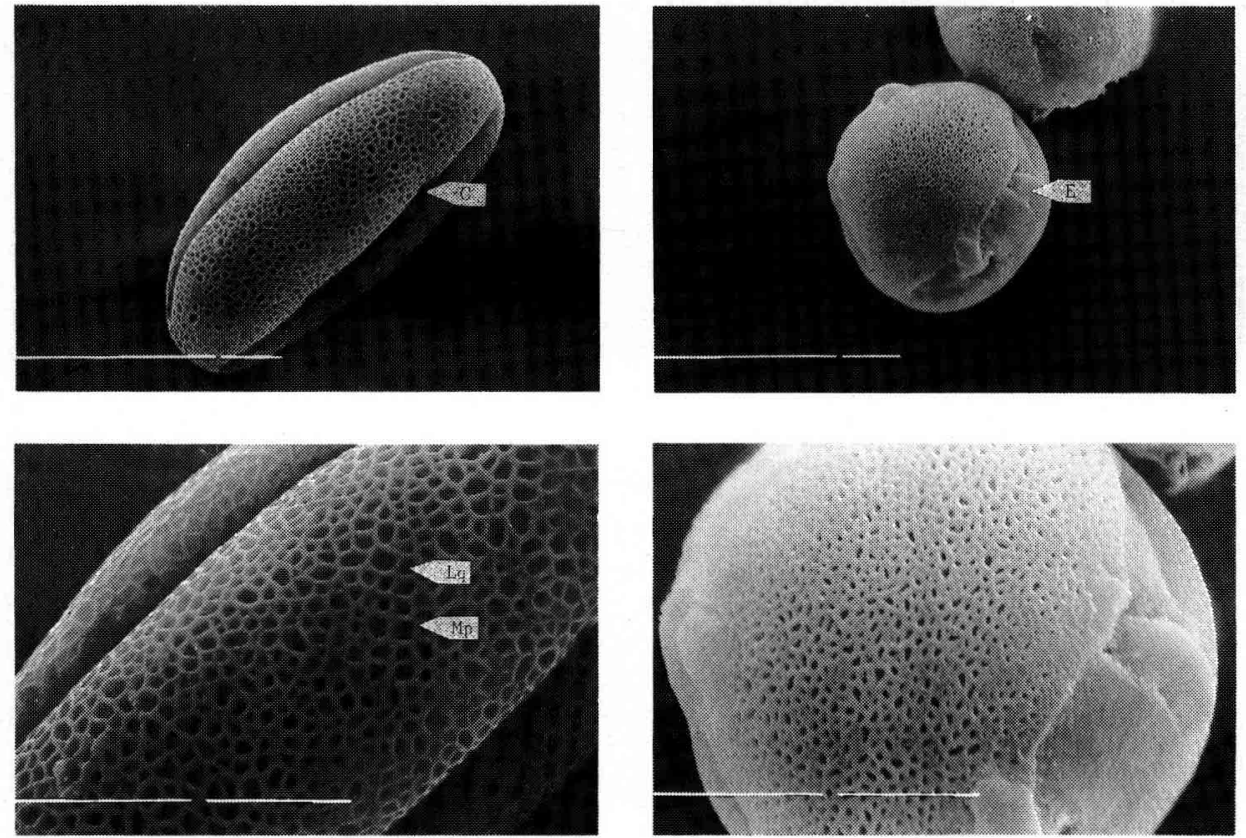

Abb 3. Pollen von Leucophyllum frutescens - Scrophulariaceae. a) kontrahiert : Form prolat oval, Colpi (C) eingezogen (x 2 000); b) expandiert : Form sphäroidal-oval, Colpi geöffnet, zwei porenähnliche Endoaperturen (E) pro Colpus sichtbar (x 2000$)$; c) kontrahiert : unregelmäßig reticulat angeordnete Muri verlaufen teilweise parallel (Mp) und bilden weite, annähernd quadratische Lumina (Lq) ( $x 5$ $000)$; d) expandiert : Muri gestaucht, Lumina stellenweise fast vollständig geschlossen ( $x 5000)$. Rechter $\mu$-Marker $=5 \mu \mathrm{m}$.

satz zum Pollen von Ligustrum vulgare engen Lumina. Auffallend sind beim kontrahierten Pollenkorn Anordnung und Form der Lumina. Sie verlaufen teilweise in längeren Bahnen aneinandergereiht, $d \mathrm{~h}$ die Muri (Mauer, die die Lumina trennen) bilden Parallelen mit Zwischenverbindungen, so daß die Lumina eine annähernd quadratische Form annehmen. Das expandierte Pollenkorn weist diese Merkmale nicht mehr so ausgeprägt auf : Durch Harmomegathie sind die Muri derart zusammengepreßt worden, daß die Lumina in einigen Bereichen fast geschlossen sind.
Die prolat-ovale Form hat sich nach der Expansion in eine sphäroidale verändert.

\section{Fagopyrum esculentum - Polygonaceae (Abb 4) :}

Die Pollen sind tricolporat. Die geöffneten Colpi zeigen größere isolierte Bacula (Stäbe). Die Skulpturierung der Ektexine ist reticulat foveolat, es liegen somit relativ kleine Luminae vor. Sie weisen eine irreguläre Gestalt mit diversen Ausbuchtungen auf, die beim kontrahierten Pollenkorn 

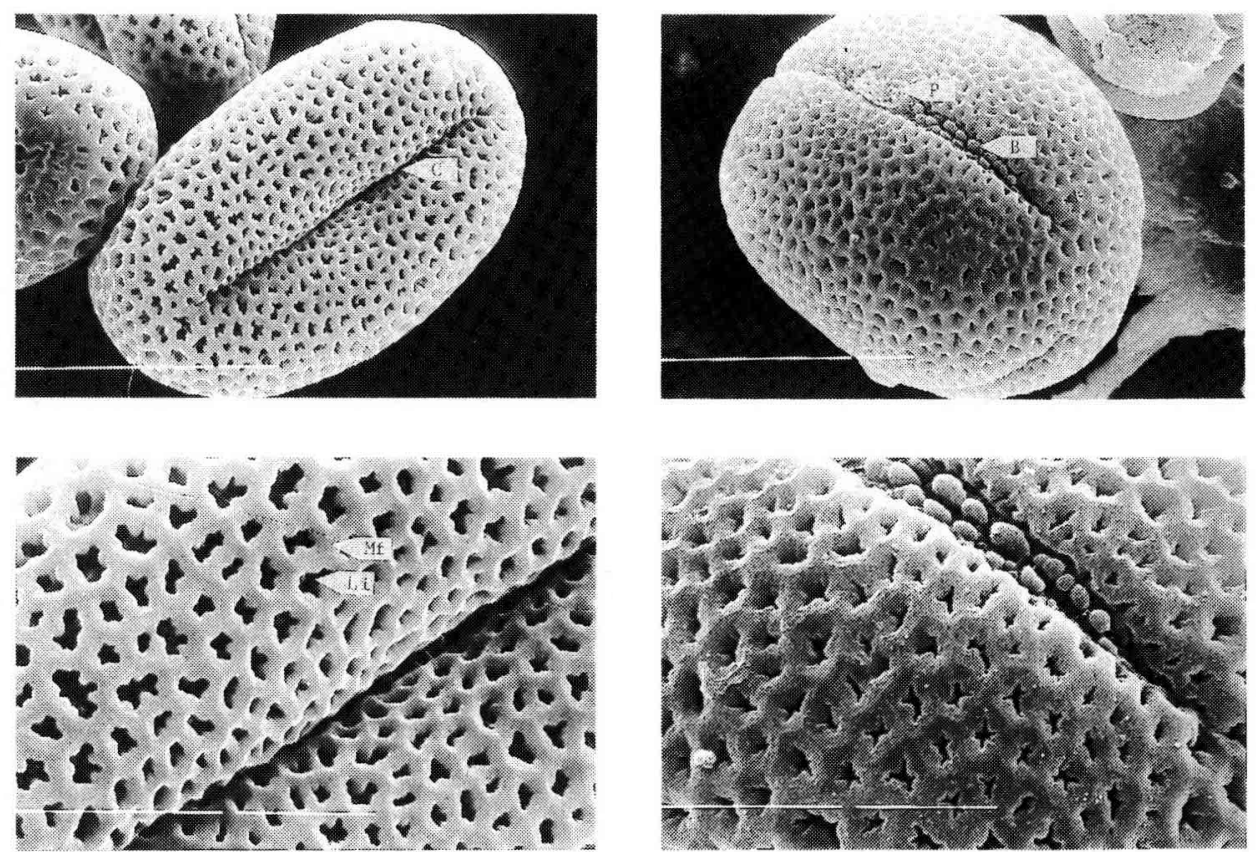

Abb 4. Pollen von Fagopyrum esculentum - Polygonaceae. a) kontrahiert : Form prolat-oval, Colpi (C) eingezogen ( $\times 2000)$; b) expandiert : Form spheroidal-oval, Colpi geöffnet, endoaperturale Pore (P) sowie Bacula (B) sichtbar ( $\times 2000)$; c) kontrahiert : reticulat-foveolat angeordnete Muri (Mf), Lumina irregulär ( $\mathrm{Li}$ ) mit rundlichen Ausbuchtungen (x 5000 ); d) expandiert : Lumina-Weite verringert, Muri gestaucht $(\times 5000)$. Rechter $\mu$-Marker $=5 \mu \mathrm{m}$.

abgerundet sind, im expandierten Zustand jedoch spitz auslaufen. Die Lumina erscheinen hier in einer sternartigen Form.

Die prolate Form des kontrahierten ovalen Pollenkornes verändert sich nach der Expansion in eine sphäroidale.

\section{Verbesina persicifolia - Compositae (Abb 5)}

Die Pollen sind tricolporat. Das perforierte Tectum trägt echinate (stachelartige) Skulpturelemente, die von einer breiten Basis spitz zulaufen. In der expandierten
Phase sind die Abstände zwischen den Basen deutlich geringer als in der kontrahierten Phase. Durch Harmomegathie hat sich die prolate Form des ovalen Pollenkornes in eine sphäroidale verändert.

\section{DISKUSSION}

Alle hier untersuchten Pollen haben die gleichen Harmomegathie-Mechanismen. Jeweils 3 ektoaperturale Colpi bewirken folgende Veränderung der Form durch die Feuchtigkeitsaufnahme des Plasmas: Prolat-ovale Formen der kontrahierten Pollen 

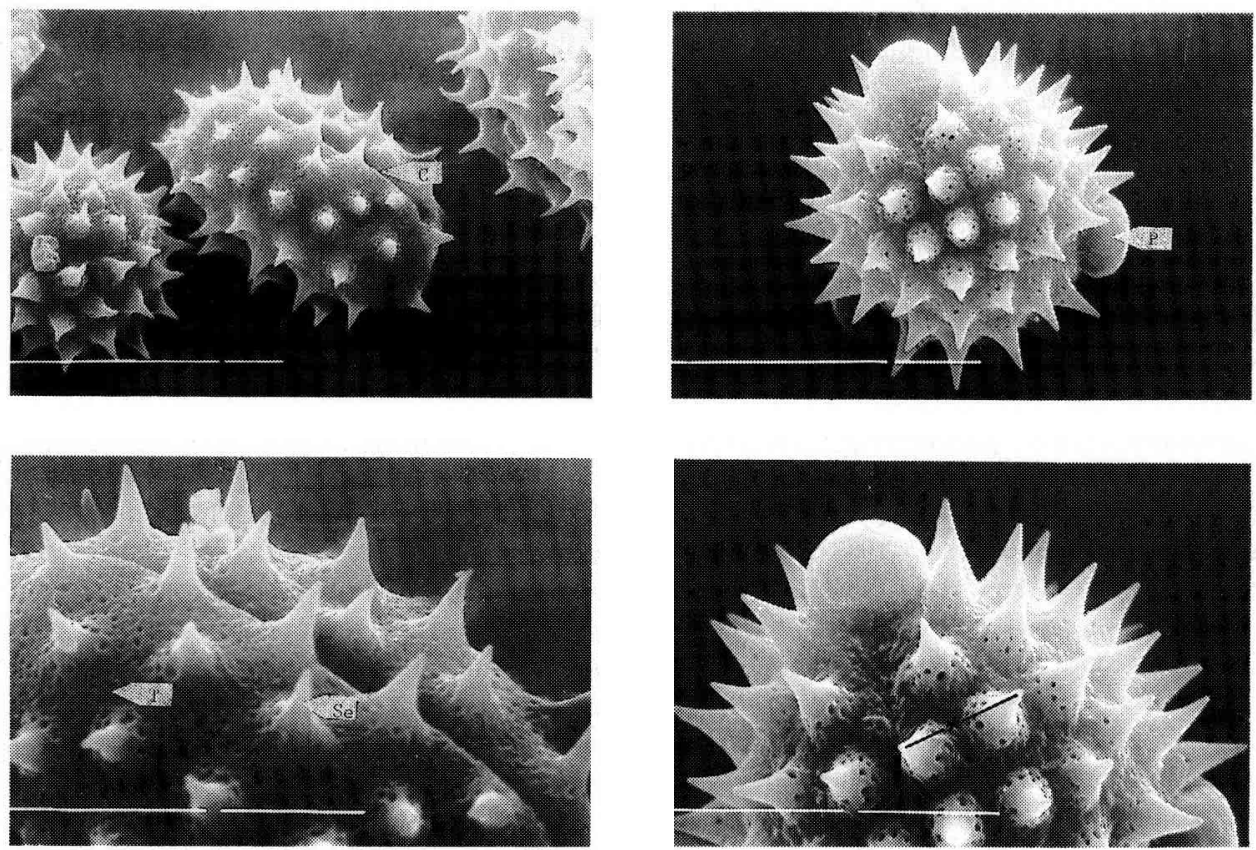

Abb 5. Pollen von Verbesina persicifolia - Compositae. a) kontrahiert : Form prolat-oval, Colpi (C) eingezogen ( $x 2000$ ); b) expandiert : Form sphäroidal-oval (hier Pol-Lage), Colpi geöffnet, endoaperturale Poren (P) sichtbar ( $\times 2000)$; c) kontrahiert : echinate Skulpturelemente (Se) auf perforiertem Tectum (T) ( $\times 5000)$; d) expandiert : deutliche Annäherung der äußeren Endpunkte der Skulpturelemente $(-*-)$. Rechter $\mu$-Marker $=5 \mu \mathrm{m}$.

werden zu sphäroidalen, $d \mathrm{~h}$ der äquatoriale Umfang vergrößert sich erheblich bei gleichzeitig enormer Verringerung der vertikalen Achse. Die durch diesen Vorgang bedingten Veränderungen der Ektexine konnten mit Hilfe des REM an striat, reticulat bzw foveolat und echinat verlaufenden Skulpturelementen festgestellt werden. Die mathematischen Grundsätze hierfür wurden von Bolick (1981) geliefert. Es bestätigte sich die in der vorangegangenen Arbeit (Dustmann und Bote, 1987) erörterte These, daß die Expansion des gesamten Pollenkornes nicht auf einer Dehnung der Intercolpien beruht. Würde ein derartiger Fall vorliegen, müßten $\mathbf{z} B$ striate Skulpturelemente eines expandiert-

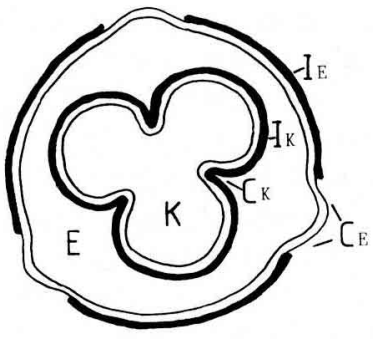

Abb 6. Schematische Darstellung eines tricolpaten Pollenkornes im Querschnitt (modifiziert nach Zander). Kontrahierte Phase $(K)$, expandierte Phase (E). Die Länge der Intercolpien $I_{K}$ und $I_{E}$ ist identisch, trotz erheblicher Vergrößerung des Umfanges. $\mathrm{CK}=$ geschlossener Colpus, $\mathrm{CE}=$ geöffneter Colpus . 
en Pollenkornes die Perforationen des Tectums deutlicher freigeben als im kontrahierten Zustand. Genau das Gegenteil ist jedoch der Fall, wie das Beispiel des Acer platanoides- Pollens zeigt (Abb 1). $\mathrm{Da}$ aber alle untersuchten Pollen eine enorme Erweiterung des äquatorialen Umfanges erfahren haben, liegt hier ein anderer Harmomegathie-Mechanismus vor. Abb 6 zeigt die schematische Darstellung eines tricolpaten Pollenkornes im Querschnitt, und zwar sowohl in der kontrahierten als auch in der expandierten Phase. Die Länge der entsprechenden Intercolpien ist identisch, lediglich ihre Form hat sich verändert. Die marginalen $\mathrm{Be}-$ reiche haben sich nach außen gebogen, die ektoaperturalen Colpi sind somit geöffnet. Am Beispiel der hier dargestellten Pollenform kann eine Erweiterung des Umfanges von ca $30 \%$ ermittelt werden, ohne daß eine Dehnung der gesamten Exine stattgefunden hat. Die weiteren Beobachtungen der verschiedenen Pollentypen haben deutlich gemacht, was bei dem oben beschriebenen Vorgang mit den Skulpturelementen geschieht.

Im kontrahierten Zustand unterliegen die Pollenkornwände einem Stress. Sie sind stark gewölbt, so daß die nach außen weisenden Skulpturelemente auseinander gebogen werden. Bei longitudinal striat verlaufenden Vallae ist dieses besonders deutlich: Perforationen auf der Basis werden freigelegt.

Verringert sich der negative Druck, läßt die Wölbung nach - eine Entspannung tritt ein. Die äußeren Bereiche der Skulpturelemente rücken näher zusammen. Eine dementsprechende Näherung ist ebenso bei den echinaten Skulpturelementen zu erkennen (Abb 5). Die Endpunkte der weit auslaufenden Skulpturelemente haben sich bei der Begradigung der Exine genähert. Verschiedene Unterarten einer reticulaten Ektexine zeigen ein abgewan- deltes Bild. Die Veränderungen beruhen aber auf dem gleichen HarmomegathieMechanismus des Intercolpiums. Abb 2, 3 und 4 belegen, daß reticulate Skulpturierungen durchaus in unterschiedlicher Weise reagieren, abhängig von der Anordnung der Muri. Da die nach außen weisenden Endungen der Skulpturelemente (in diesem Fall die auf den Columellae befindlichen Muri) in einem vernetzten Verbund stehen, findet eine Stauchung statt.

Alle Pollen, die in der Melissopalynologie von Bedeutung sind, unterliegen der Harmomegathie. Es handelt sich hierbei um lebende, nicht um fossile Pollen. Letztere besitzen keinen plasmatischen Inhalt mehr und verändern somit in überschaubaren Zeiträumen weder Form noch Volumen. Die Pollen des Honigs unterliegen jedoch den in dieser Arbeit beschriebenen Variablen. Zur Beurteilung von Honigen mit speziellen Fragestellungen ist es unerläßlich, auch scheinbar gleichstrukturierte Pollen zu differenzieren. Der Vergleich der Exine kontrahierter und expandierter Pollen mit Hilfe des RasterElektronenmikroskopes ermöglicht im $\mathrm{Ge}$ gensatz zum Lichtmikroskop diese weitergehende Differenzierung. Um Fehlinterpretationen auszuschließen, ist das Verständnis für das Zustandekommen der momentanen Charakteristik der Pollengestalt von besonderer Bedeutung für die Melissopalynologie.

\section{Summary - Scanning electron micro- scopic studies of pollen from honey. III. The harmomegathy-mechanism and its effect on the exine-structure using dif- ferent pollen types. Pollen grains found in honey are subjected to changes in vol- ume and shape by hydration of the plasm - the harmomegathy - which enable the pollen grain to harmonize with the sur- rounding forces. At dehiscence from the}


anther into the dehydrating atmosphere, pollen grains change their shape so that the apertures close (state of moisture stress = contraction). Conversely, the exine relaxes by hydration when the pollen is on the stigma of the blossom or has fallen into nectar. In addition to volume and shape, the surface pattern of the intercolpium may also change by harmomegathy. Pollen with different sculpturing types were analyzed by SEM in states of contraction and expansion. Contracted pollen grains were transferred into filtered honey for expansion and prepared for SEM after isolation into a wire screen. All tricolpate pollen grains were found to change their prolateoval into a spheroidal shape by harmomegathy. The analyzed sculpturing patterns (striate, reticulate, foveolate, echinate) show that there is no stretching of the general intercolpium. If such a stretching occurred, the striate sculpturing element should show tectate perforations more visible than in a state of contraction. However, fig 1 shows the opposite : In expanded pollen grains of Acer platanoides, the tectum is closed by supratectate valla. Fig 6 illustrates that the enlargement of the pollen circumference does not require a general stretching, but only a bending of the intercolpium which simultaneously opens the apertures. Due to this harmomegathy mechanism, the external regions of the sculpturing elements draw close as on the pollen of Verbesina persicifolia with its particularly elongated echinate elements (fig 5). Similarly, the expanded pollen of Ligustrum vulgare shows a lumina width decrease of the reticulate pattern (fig 2), and the Leucophyllum frutescens pollen an additional compressive strain of the muri (fig 3). The foveolate sculpturing elements on the Fagopyrum esculentum pollen undergo a compression important enough to induce considerable changes in lumina shape. The knowledge of pollen surface changes described in this study should facilitate the melissopalynological identification of pollen using SEM.

\section{pollen / honey / harmomegathy / exine structure / SEM}

Résumé - Étude du pollen du miel en
microscopie électronique à balayage. III. Le mécanisme d'harmomégathie et son action sur la structure de l'exine chez divers types polliniques. Les pollens présents dans le miel sont susceptibles de changer de volume et de forme par hydradation ou déshydratation du cytoplasme : c'est l'harmomégathie, qui permet au grain de pollen de se mettre en harmonie avec les forces environnantes. A la déhiscence de l'anthère en atmosphère déshydratante les grains de pollen ferment leurs apertures, ce qui induit un changement de forme (état de stress = contraction). Inversement, l'exine se relâche par hydratation lorsque le pollen est sur le stigmate de la fleur ou qu'il est tombé dans le nectar. Outre le volume et la forme, l'harmomégathie modifie les structures superficielles de l'intercolpium. Nous avons analysé au microscope électronique à balayage (MEB) des grains de pollen présentant divers types de sculptures, en état de contraction et d'expansion. Les grains de pollen contractés ont été mis dans du miel filtré pour passer en état d'expansion, isolés dans un filet métallique, puis préparés pour le MEB. Tous les grains de pollen tricolpés ont modifié leur forme prolateovale en forme sphéroïdale par harmomégathie. Les sculptures analysées (striée, réticulée, fovéolée, échinulée) montrent qu'il n'y a aucun allongement (extension) de l'intercolpium général. Si tel était le cas, les éléments de sculpture striée devraient avoir des perforations du tectum plus visibles qu'en état de contraction. La figure 1 montre le contraire : chez les grains de pollen d'Acer platanoides en état d'expan- 
sion, le tectum est fermé par les vallées supratectées. La figure 6 montre que l'agrandissement de la circonférence du pollen ne nécessite pas l'extension générale de l'intercolpium, mais seulement sa flexion, qui ouvre en même temps les apertures. A cause des mécanismes d'harmomégathie, les régions externes des éléments de sculptures se rapprochent, comme on le voit avec le pollen de Verbesina persicifolia et ses éléments échinulés particulièrement allongés (fig 5). De la même façon le pollen en état d'expansion de Ligustrum vulgare montre une diminution de la largeur des lumières de la sculpture réticulée (fig 2) et le pollen de Leucophyllum frutescens une compression des murs (fig 3). Les éléments de sculpture fovéolée du pollen de Fagopyrum esculentum subissent une telle compression que la forme des lumières change considérablement (fig 4). La connaissance des changements de la surface du pollen décrits ici, devrait être utile à l'identification des pollens en MEB dans le cadre de la mélissopalynologie.

\section{Pollen / miel / harmomégathie / struc- ture de l'exine / MEB}

\section{LITERATUR}

Blackmore S, Barnes SH (1985) Harmomegathic mechanisms in pollen grains. In: Pollen and Spores: Form and Function. No 12 (Blackmore S, Ferguson IK, eds) 137-149

Bolick MR (1981) Mechanics as an aid to interpreting pollen structure and function. Rev $\mathrm{Pa}$ laeobot Palynol 35, 61-79
Dustmann JH, Bote K (1985) Raster-Elektronenmikroskopische Studien an Bienenhonig. I. Vergieich verschiedener Präparationsmethoden. Apidologie 16, 331-340

Dustman JH, Bote K (1987) Raster-Elektronenmikroskopische Studien an Bienenhonig. II. Vergleich der Exine-Strukturen trockener und in Honig expandierter Pollen bei verschiedenen Apfel- bzw. Zierapfelsorten. Gartenbauwissenschaft 52, 271-278

Erdtman G (1952) Pollen Morphology and Plant Taxonomy - Angiosperms. Almquist \& Wiksells, Uppsala

Faegri K, Iversen J (1964) Textbook of Pollen Analysis. Scandinavian University Books, Munksgaard, Copenhagen

Iwanami Y, Sasakuma T, Yamada Y (1988) Pollen: Illustrations and Scanning Electronmicrographs. Springer Verlag, Berlin. Heidelberg, New York

Payne WW (1972) Observations of Harmomegathy in Pollen of Anthophyta. Grana 12, 93-98

Payne WW (1981) Structure and function in angiosperm pollen wall evolution. Rev Palaeobot Palynol 35, 39-59

Punt W (1986) Functional factors influencing pollen form. In: Pollen and Spores: Form and Function, No 12 (Blackmore S, Ferguson IK, eds), 97-101

Suarez-Cervera M, Seoane-Camba JA (1986) On the exine elasticity in the Lavendula dentata $L$ pollen grains. In: Pollen and Spores: Form and Function. No 12 (Blackmore S, Ferguson IK, eds), 409-411

Wodehouse RP (1935) Pollen Grains. McGrawHill, New York

Zander E (1935) Beiträge zur Herkunftsbestimmung bei Honig. I. Pollengestaltung und Herkunftsbestimmung bei Blütenhonig. Verlag der Reichsfachguppe Imker e V, Berlin 